\title{
Hydrophobic effect of silica functionalized with silylated Ti-salicylaldimine complex on limonene oxidation by aqueous hydrogen peroxide
}

\author{
LAI SIN YUAN ${ }^{\mathrm{a}}$, SHEELA CHANDREN ${ }^{\mathrm{a}}$, JON EFENDI ${ }^{\mathrm{b}}, \mathrm{CHIN}^{\mathrm{S}}$ SIONG HO ${ }^{\mathrm{c}}$ and \\ HADI NUR ${ }^{\mathrm{a}, \mathrm{d}, *}$ \\ ${ }^{a}$ Centre for Sustainable Nanomaterials, Ibnu Sina Institute for Scientific and Industrial Research, \\ Universiti Teknologi Malaysia, 81310 UTM Skudai, Johor, Malaysia \\ ${ }^{b}$ Department of Chemistry, Faculty of Mathematics and Science, Universitas Negeri Padang, \\ Padang 25131, Indonesia \\ ${ }^{c}$ Low Carbon Asia Research Center, Faculty of Built Environment, Universiti Teknologi Malaysia, \\ 81310 UTM Skudai, Johor, Malaysia \\ ${ }^{\mathrm{d}}$ Department of Physics, Institut Sains dan Teknologi Nasional, Jl. Moh. Kahfi II, Jagakarsa, \\ Jakarta Selatan 12640, Indonesia \\ e-mail: hadi@kimia.fs.utm.my
}

MS received 27 May 2015; revised 14 July 2015; accepted 2 August 2015

\begin{abstract}
This research work describes the effect of hydrophobicity in inducing the diffusion of alkene substrates to the catalytic active sites. The aim of this research is to improve the catalytic activity by tailoring the degree of hydrophobicity of the catalyst. Silica functionalized with solid non-silylated Ti-salicylaldimine complex was prepared at room temperature by mixing imine ligand and Ti(IV) sulphate solution. The amorphous, solid complex formed was further silylated with octadecyltrimethoxysilane (OTMS) to induce hydrophobicity. The composition of the resulting silica functionalized with silylated Ti-salicylaldimine complex was varied with Ti:OTMS molar ratio in the range of 1:1/4 to $1: 4$ at room temperature. The successful attachment of alkylsilyl groups to silica functionalized with Ti-salicylaldimine complex was proven by the FTIR and ${ }^{29} \mathrm{Si}$ solid state NMR spectra. The FTIR spectra showed increasing peak area for $\mathrm{sp}^{3} \mathrm{C}-\mathrm{H}$ stretching mode (ca. $2919 \mathrm{~cm}^{-1}$ and $2850 \mathrm{~cm}^{-1}$ ) and decreasing peak area for Si-OH band with increasing amount of OTMS. TGA showed less water content with higher amount of alkylsilyl groups in the catalyst. This is in agreement with the lower kinetic rate of water adsorption capacity for the hydrophobic catalysts prepared. It was observed that the hydrophobic, silica functionalized with silylated Ti-salicylaldimine complex exhibited higher substrate conversion and reusability compared to the non-silylated catalyst.
\end{abstract}

Keywords. Hydrophobicity; silylation; silica functionalized with silyated Ti-salicylaldimine complex; limonene oxidation; octadecyltrimethoxysilane.

\section{Introduction}

Numerous efforts have been made to improve the performance of heterogeneous catalysts in various reactions. One of the factors for such improvement is the local coordination of the active sites, for instance, tetrahedral local structure of titanium(IV), which is a well-known catalytic active site for the epoxidation and oxidation of organic compounds. ${ }^{1,2}$ Although Titanium Silicalite-1 (TS-1) can achieve high catalytic selectivity in epoxidations, ${ }^{1}$ it is microporous and hence, many

\footnotetext{
*For correspondence
}

cyclic, branched and long chain olefins cannot diffuse into the pores to react with the active sites. Thus, mesoporous or polyoxometalate Ti-containing catalysts, such as $\mathrm{TiO}_{2}$-zeolite, ${ }^{3} \mathrm{Ti}-\mathrm{SBA}-15,{ }^{4} \mathrm{Ti}-\mathrm{POM},{ }^{5} \mathrm{Ti}-$ MCM- $41^{6}$ and others, which can accommodate larger size of substrates have been synthesized. Apart from that, other approaches have been carried out as well i.e., modification of surface properties of heterogeneous catalysts, such as the sulphation of TS- $1,{ }^{7}$ fluorinated $\mathrm{TiO}_{2}-\mathrm{ZrO}_{2},{ }^{8} \quad \mathrm{Nb}_{2} \mathrm{O}_{5} / \mathrm{TS}-1,{ }^{9}$ silylation of several catalysts ${ }^{8,13-18}$ and many others, in order to improve the catalytic activity.

In this research, we focused on the surface modification of heterogeneous catalysts. Of late, surface modification has been widely utilized as it could solve a 
number of problems, such as leaching and metal poisoning. Previous studies have proven that the catalytic activity of catalysts containing Ti(IV) can be greatly enhanced for bulky and long chain organic compounds. However, the leaching of Ti(IV) active sites still can occur ${ }^{10}$ and the efficiency of hydrogen peroxide $\left(\mathrm{H}_{2} \mathrm{O}_{2}\right)$ decreases ${ }^{11}$ in organic-aqueous oxidation. Support materials, i.e. silica, contain high density of silanol groups $(\mathrm{SiOH})$ which is highly hydrophilic. The water molecules produced from $\mathrm{H}_{2} \mathrm{O}_{2}$ easily form strong hydrogen bonds with $\mathrm{Si}-\mathrm{OH}$ and compete with $\mathrm{H}_{2} \mathrm{O}_{2} .{ }^{12,13}$ Apart from that, water molecules are able to open the oxirane ring of epoxides to form diol, which can strongly adsorb to the titanium(IV)'s active sites, causing catalyst deactivation. ${ }^{12,13}$ The catalytic results were significantly affected and thereby, some researchers ${ }^{8,13-18}$ have come out with various silylating agents to be grafted or attached to the surface of catalyst to induce hydrophobicity.

Previously, it was reported that the long hydrophobic chain of octadecyltrichlorosilane (OTS), which was attached to fluorinated- $\mathrm{TiO}_{2}-\mathrm{ZrO}_{2}\left(\mathrm{~F}-\mathrm{TiO}{ }_{2}-\mathrm{ZrO}_{2}\right)$, managed to achieve higher TON for titanium(IV) active sites as compared to $\mathrm{F}-\mathrm{TiO}_{2}-\mathrm{ZrO}_{2}$ in 1-octene oxidation by aqueous $\mathrm{H}_{2} \mathrm{O}_{2} .{ }^{8}$ Another research showed that the short hydrophobic chain of N-methyl-N-(trimethylsilyl)trifluoroacetamide (MSTFA) has also increased the epoxide yields in the epoxidation of cyclohexene with $\mathrm{H}_{2} \mathrm{O}_{2} \cdot{ }^{14}$ Several researches ${ }^{13,15-18}$ showed that catalysts with surface modification of hydrophobic silylated titanium(IV) catalysts performed remarkable higher activity than the non-silylated counterpart of titanium(IV) catalysts. These examples proved that the surface properties ${ }^{13-18}$ of catalysts play significant roles in achieving high catalytic activity and selectivity.

Herein, this research highlights the types of silylating agents and catalysts utilized in the oxidation of small aromatic alkene. Most of the silylating agents used in previous reports were of short alkylsilyl chain with trimethylsilyl and hexamethylsilyl groups in the oxidation of small alkenes by aqueous $\mathrm{H}_{2} \mathrm{O}_{2}$. To date, the findings of silylation with long alkylsilyl chain are lessj reported. Besides that, catalysts commonly used to improve hydrophobicity are metal oxides, especially silylated Ti-MCM-41 and silylated Ti-MCM-48. ${ }^{12-14,17}$ Thus, an attempt to produce a different type of catalyst, i.e. silica functionalized with silylated Ti-salicyladimine complex is shown here. A series of variations in the amount of octadecylsilyl $\left(\mathrm{C}_{18}\right)$ group is used to silylate Ti-salicylaldimine complex for the oxidation of limonene (small, aromatic alkene) by aqueous $\mathrm{H}_{2} \mathrm{O}_{2}$.

\section{Experimental}

\subsection{Materials}

The chemicals used are salicylaldehyde (98\%, SigmaAldrich), absolute ethanol (Merck), 3-aminopropyltrimethoxysilane (97\%, Sigma-Aldrich), titanium(IV) sulphate solution (24\%, Kanto Chemical Co., Inc.), and octadecyltrimethoxysilane (90\%, Acros Organics) and molecular sieves $3 \AA ̊$ ( 25 g, Sigma-Aldrich).

\subsection{Methods}

2.2a Synthesis of silica functionalized with nonsilylated Ti-salicylaldimine complex: Silica functionalized with Ti-salicylaldimine complex was synthesized as follows: first, salicylaldimine ligand, which is generally known as Schiff base ligand, was prepared under nitrogen flow. Salicylaldehyde $(0.05 \mathrm{~mol}$, $5.30 \mathrm{~mL})$ in absolute ethanol $(20.00 \mathrm{~mL})$ and 3aminopropyltrimethoxysilane $(0.05 \mathrm{~mol}, 9.30 \mathrm{~mL})$ in absolute ethanol $(20.00 \mathrm{~mL})$ were added drop-wised into a three-neck round bottom flask containing molecular sieves $3 \AA$ ( $25 \mathrm{~g})$ and absolute ethanol $(50.00 \mathrm{~mL})$. Salicylaldehyde $98 \%(0.05 \mathrm{~mol}, 5.3 \mathrm{~mL})$ reacted with 3 -aminopropyltrimethoxysilane $(0.05 \mathrm{~mol}, 9.3 \mathrm{~mL})$ to form bright yellowish ligand under mild stirring at room temperature for a few minutes. The mixture was centrifuged at $4000 \mathrm{rpm}$ for $5 \mathrm{~min}$ and then vacuumed to remove the ethanol solvent. Dark yellowish ligand was formed after the removal of the solvent. Titanium(IV) sulphate solution $(0.01 \mathrm{~mol}, 8.9 \mathrm{~mL})$ in $10.0 \mathrm{~mL}$ of absolute ethanol was added into the yellowish ligand (0.02 mol) in $40.0 \mathrm{~mL}$ absolute ethanol. The solution quickly turned into colloidal yellowish suspension. It was stirred overnight at room temperature to complete the reaction. The resulting powder was centrifuged at $4000 \mathrm{rpm}$ for $10 \mathrm{~min}$ and washed with ethanol, followed by hexane, twice each. The amorphous complex formed was dried at room temperature for a few days. The weight of the silica functionalized with nonsilylated Ti-salicylaldimine complex was $0.58 \mathrm{~g}$ with $24 \%$ of titanium(IV) in the catalyst.

$2.2 \mathrm{~b}$ Silica functionalized with silylated of $\mathrm{Ti}$ salicylaldimine complex: The silylation of Tisalicylaldimine complex by octadecyltrimethoxysilane (OTMS) with Ti:OTMS molar ratio of 1:4 was carried out as follows: Ti-salicylaldimine complex (10.00 mmol) was added with OTMS $(2.50 \mathrm{mmol}, 1.18 \mathrm{~mL})$ and dissolved in toluene $(10.00 \mathrm{~mL})$. The mixture was then stirred overnight. To prevent hydrolysis of 
OTMS, the preparation process was done in a glove box. A similar procedure was also carried out to for the silylation of Ti-salicylaldimine complex with different Ti:OTMS molar ratio $(1: 1 / 3,1: 1 / 2,1: 1,1: 2,1: 3$, and $1: 4)$. The solid catalysts were centrifuged, followed by washing with ethanol, toluene and hexane, twice each.

The concentration of Ti(IV) sulphate solution was maintained at $0.01 \mathrm{~mol}$ for the entire synthesis process, regardless of the OTMS concentration.

\subsection{Characterizations}

The solid catalysts obtained were characterized by FTIR, diffuse reflectance UV-Vis spectroscopy (DR UV-Vis), ${ }^{29} \mathrm{Si}$ solid-state magic angle spinning NMR $\left({ }^{29} \mathrm{Si}\right.$ MAS NMR) spectroscopy, scanning electron microscopy (SEM) and thermogravimetric analysis (TGA). All samples were preheated at $60^{\circ} \mathrm{C}$ for a few hours to remove any adsorbed water. In order to confirm the successful removal of water content in the samples, the weight of the samples were measured before and after heating until a constant weight of samples was obtained. There were no fixed heating hours because each of the catalysts gave their constant weight at different times according to their degree of silylation. It was observed that shorter time was required for catalysts with higher coverage degree of hydrophobic surface with alkylsilyl groups compared to the more hydrophilic surface with lower coverage degree of alkylsilyl groups.

FTIR spectra were collected on a Perkin Elmer Spectrum One spectrometer with 10 scans and resolution of $4 \mathrm{~cm}^{-1}$, in the range of $4000-400 \mathrm{~cm}^{-1}$. Potassium bromide $(\mathrm{KBr})$ pellet technique was used to examine the functional groups of a series of silica functionalized silylated Ti-salicyladimine complex (solid), where the samples were mixed with $\mathrm{KBr}$ in the weight ratio of $1: 100$. The DR UV-Vis analysis was recorded by Perkin Elmer Ultraviolet-visible Spectrometer Lambda 900 and plotted using Kubelka-Munk function, in the range of 200-800 $\mathrm{nm}$, using barium sulphate as the standard. Meanwhile, the ligand (liquid) was recorded in the range of 190-800 nm, using ethanol as the reference. The ${ }^{29} \mathrm{Si}$ solid state MAS NMR experiments were performed using Bruker Avance $400 \mathrm{MHz} 9.4 \mathrm{~T}$ spectrometer. The spectra were recorded at $79.44 \mathrm{MHz}$ using $4 \mu$ s radio frequency pulses, a recycle delay of $60 \mathrm{~s}$ and spinning rate of $7.0 \mathrm{kHz}$ using a $4 \mathrm{~mm}$ zirconia sample rotor. ${ }^{29} \mathrm{Si}$ MAS NMR chemical shifts were referred to external TMS at $0 \mathrm{ppm}$. The morphology of the solid catalyst was observed using SEM. The amount of organic moiety in the catalysts was determined by Thermogravimetric Analyzer Mettler Toledo using platinum crucible.

\subsection{Catalytic activity of silica functionalized with non-silylated and silylated Ti-salicylaldimine complexes}

The catalytic testing was carried out at $70^{\circ} \mathrm{C}$ with (R)(+)-Limonene (5.00 mmol, $0.83 \mathrm{~mL}), 30 \% \mathrm{H}_{2} \mathrm{O}_{2}(0.01$ $\mathrm{mol}, 0.38 \mathrm{~mL})$ and the catalyst $(0.05 \mathrm{~g})$ for $22 \mathrm{~h}$ under magnetic stirring. The products of the reactions were analyzed by Gas Chromatography, Shimadzu model GC-2014 equipped with BPX-5 column (30 m length, $0.25 \mathrm{~mm}$ inner diameter, $0.25 \mu \mathrm{m}$ film thickness) and a flame ionization detector (FID). The temperature program of the column oven was performed with the initial temperature of $60^{\circ} \mathrm{C}$, heating rate of $10^{\circ} \mathrm{C} / \mathrm{min}$ and the temperature was maintained at $100^{\circ} \mathrm{C}$ for $1 \mathrm{~min}$. After that, the same heating rate $\left(10^{\circ} \mathrm{C} / \mathrm{min}\right)$ was applied till $138^{\circ} \mathrm{C}$ and then maintained for $2 \mathrm{~min}$. Following that, the heating rate was increased to $15^{\circ} \mathrm{C} / \mathrm{min}$ by heating up to $150^{\circ} \mathrm{C}$ and maintained for $1 \mathrm{~min}$ to elute the products left inside the column.

Limonene (organic phase) and aqueous $\mathrm{H}_{2} \mathrm{O}_{2}$ (aqueous phase) are an immiscible liquid-liquid system. In the reaction process, stirring was required to remove the phase-boundary between limonene and aqueous $\mathrm{H}_{2} \mathrm{O}_{2}$, in order to form one homogeneous phase. After the reaction has been stopped, the liquids separated back as two phases. The upper organic phase containing the products (carveol, carvone, epoxides, etc.) was separated and taken for gas chromatography analysis. Undeniably, some products might have dissolved in the aqueous phase, but the amount is insignificant because the products are all nonpolar liquids. Thus, the products possess higher solubility in the organic phase compared to the aqueous phase. Leaching was not tested in this experiment as this research serves as a preliminary experiment to investigate the hydrophobicity effects towards limonene oxidation reaction. Further investigation will be carried out for the leaching in future work.

\section{Results and Discussion}

\subsection{Physical properties}

Figure 1 shows the FTIR spectra of silica functionalized with non-silylated Ti-salicylaldimine complex and silylated Ti-salicylaldimine complex functionalized silica by octadecyltrimethoxysilane (OTMS) with different Ti:OTMS molar ratios. 




Figure 1. FTIR spectra of (a) salicylaldimine ligand, (b) silica functionalized with Ti-salicylaldimine complex, and silica functionalized with Tisalicylaldimine complex silylated with Ti/OTMS molar ratio of (c) 1:1/4, (d) $1: 1 / 3$, (e) $1: 1 / 2$, (f) $1: 1$, (g) $1: 2$, (h) $1: 3$, (i) $1: 4$.

It is worth noting that all the spectra showed two absorption peaks at $601 \mathrm{~cm}^{-1}$ and $697 \mathrm{~cm}^{-1}$, corresponding to the Ti-N and Ti-O bonds, respectively. ${ }^{19,20}$ The band at $1610 \mathrm{~cm}^{-1}$ (figure 1 (b) - (i)) is assigned to $\mathrm{C}=\mathrm{N}$ stretching mode. The shifting of free azomethine group (figure 1 (a)) from higher frequency of ca. $1633 \mathrm{~cm}^{-1}$ to lower frequency of $1610 \mathrm{~cm}^{-1}$ is caused by the partial attraction of electron density in $\mathrm{C}=\mathrm{N}$ towards $\mathrm{Ti}^{4+}$ ion. This indicates that $\mathrm{Ti}(\mathrm{IV})$ has been successfully coordinated with azomethine nitrogen atom, ${ }^{21,22}$ which resulted in the weakening of the azomethine group bonding. The strong absorption peaks that appeared at $c a .1127 \mathrm{~cm}^{-1}$ and 1038 $\mathrm{cm}^{-1}$ (figure 1 (b) - (i)) are typically attributed to the asymmetric and symmetric stretching bands of $\mathrm{Si}-\mathrm{O}-\mathrm{Si}$, respectively.

Nevertheless, the phenolic oxygen bonded to $\mathrm{Ti}^{4+}$ also exhibited frequency in the same range as that of the symmetric stretching of Si-O-Si, which was at $1050-$ $1100 \mathrm{~cm}^{-1} .^{23}$ This occurrence assisted in reinforcing the argument that the complex containing $\mathrm{Ti}^{4+}$ is bonded to the oxygen of the phenolic group instead of Si-O-Ti bonds, as no peak at $c a .960 \mathrm{~cm}^{-1}$ can be detected. ${ }^{24}$ The absorption band of $\mathrm{Ti}^{4+}$ coordinated with phenolic oxygen anion $\left(\mathrm{C}_{6} \mathrm{H}_{5} \mathrm{O}^{-}\right)$was less evident because it overlapped with $\mathrm{Si}-\mathrm{O}-\mathrm{Si}$ symmetric stretching. It is suggested that the $\mathrm{Si}-\mathrm{OH}$ bands located at $c a .995 \mathrm{~cm}^{-1}$ have higher frequency than those previously reported ${ }^{24}$ because Ti(IV) ion was not bonded to Si-O. The trend of Si-OH bands $\left(995 \mathrm{~cm}^{-1}\right)$ decreased with increasing concentration of OTMS (figure 1 (c) - (i)).

The peak areas of SiOH/Ti(IV), denoted as Peak Area $_{[995]} /$ Peak Area $_{[602]}$, decreased in the order of low to high concentration of silica functionalized with silylated Ti-salicylaldimine complex. This is due to the hydrogen atoms from $\mathrm{OH}$ groups that have been replaced by long chain $\mathrm{Si}-\mathrm{C}_{18} \mathrm{H}_{38} \cdot{ }^{14,25}$ On the contrary, the intensity of asymmetric and symmetric $\mathrm{sp}^{3} \mathrm{C}-\mathrm{H}$ stretching displayed at $c a .2919$ and $2850 \mathrm{~cm}^{-1}$, respectively, increased with the amount of OTMS. This indicates that OTMS has been successfully silylated on the surface of Ti-salicylaldimine complex. The FTIR results listed in the table 1 show the areas of $\mathrm{C}-\mathrm{H}$ and Ti$\mathrm{N}$ bands, [Peak Area ${ }_{[2919,2850]} /$ Peak Area $\left.{ }_{[602]}\right]$. In addition, there are two other prominent peaks that can be observed at $c a$. 1469 and $720 \mathrm{~cm}^{-1}$ in the FTIR spectra. These two important bands originated from $\mathrm{CH}_{2}$ scissoring and $\mathrm{CH}_{3}$ rocking modes, respectively. ${ }^{14}$ All the above phenomena indicate that OTMS has been successfully anchored on the complexes and hence, led to the enhancement in the hydrophobicity of the catalysts. 
Table 1. FTIR peak area of silica functionalized with nonsilylated and silylated Ti-salicyladimine complex with different amounts of octadecyltrimethoxysilane (OTMS) ${ }^{\mathrm{a}}$.

Ti-salicylaldimine complex with the molar ratio of Ti(IV):OTMS

Ratio of Peak area $\mathrm{b}_{[2850 ; 2919]}^{\mathrm{b}} /$ Peak area ${ }_{[602]}^{\mathrm{c}}$

Ti:OMS 1:1/4

1.03

Ti:OMS $1: 1 / 3$

1.29

Ti:OMS $1: 1 / 2$

1.32

Ti:OMS 1:1

1.90

1.98

2.86

Ti:OMS 1:3

4.67

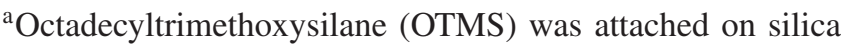
functionalized with Ti-salicylaldimine complex in order to prove that the peak area of $\mathrm{C}-\mathrm{H}$ stretching mode increased with increasing concentration of OTMS.

${ }^{\mathrm{b}}$ The peak area of symmetric and asymmetric $\mathrm{sp}^{3} \mathrm{C}-\mathrm{H}$ stretching bands from OTMS can be seen at ca. 2919 and $2850 \mathrm{~cm}^{-1}$, respectively.

${ }^{\mathrm{c}}$ The absorption band at $602 \mathrm{~cm}^{-1}$ is attributed to Ti-N from silylated Ti-salicylaldimine complex.

\subsection{Diffuse reflectance UV-Visible analysis}

Figure 2 shows the diffuse reflectance UV-Vis spectra of (a) salicylaldimine ligand, (b) silylated salicylaldimine ligand, (c) silica functioanlized with Ti-salicylaldimine complex and (d) silica functioanlized with silylated
Ti-salicylaldimine complex. The bands of salicylaldimine ligand (figure 2(a)) at 227 and $248 \mathrm{~nm}$ can be assigned to the $\pi-\pi *$ transition of benzene ring and imine group $(\mathrm{C}=\mathrm{N})$, respectively. The bands at lower energy of $c a .314$ and $402 \mathrm{~nm}$ were attributed to the substituted phenol group $\left(\mathrm{C}_{6} \mathrm{H}_{5} \mathrm{O}^{-}\right)$ and imine group $(\mathrm{C}=\mathrm{N})$ with $\mathrm{n}-\pi^{*}$ transitions. The absorption bands of silylated salicylaldimine ligand (figure 2(b)) showed similar pattern as those of salicylaldimine ligand (figure 2(a)). It is observed that a small peak appeared at $270-280 \mathrm{~nm}$, which can be attributed to the ethanol solvent's cut-off. This is because salicylaldimine ligand is polar and it might have induced the shifting of ethanol polar solvent from shorter wavelength, i.e. $210 \mathrm{~nm}$, to longer wavelength, $c a .270 \mathrm{~nm}$. In the spectra of the complexes (figure 2(c) and (d)), a shoulder band in the range of 200-250 $\mathrm{nm}$ can be assigned to the association of the $\pi-\pi *$ transitions of aromatic ring and azomethine group.

Laporte-allowed LMCT of oxygen from phenyl group $\left(\mathrm{C}_{6} \mathrm{H}_{5} \mathrm{O}^{-}\right)$to metal $\left(\mathrm{Ti}^{4+}\right)$ can be proven by the shift from $315 \mathrm{~nm}$ to $300 \mathrm{~nm}$ after the complexation $\left(\mathrm{C}_{6} \mathrm{H}_{5} \mathrm{O}^{-} \mathrm{Ti}^{4+}\right) .{ }^{26}$ It is observed that the $\mathrm{n}-\pi *$ of imine group, $\mathrm{C}=\mathrm{N}$, has shifted to lower frequency due to the coordination of imine nitrogen to metal $\left(\mathrm{C}=\mathrm{NTi}^{4+}\right)$ to form complexes. ${ }^{22,27}$ The UV spectra are broader and stronger in the range of $350-500 \mathrm{~nm}$ for the silica functionalized with silylated Ti-salicyladimine complex

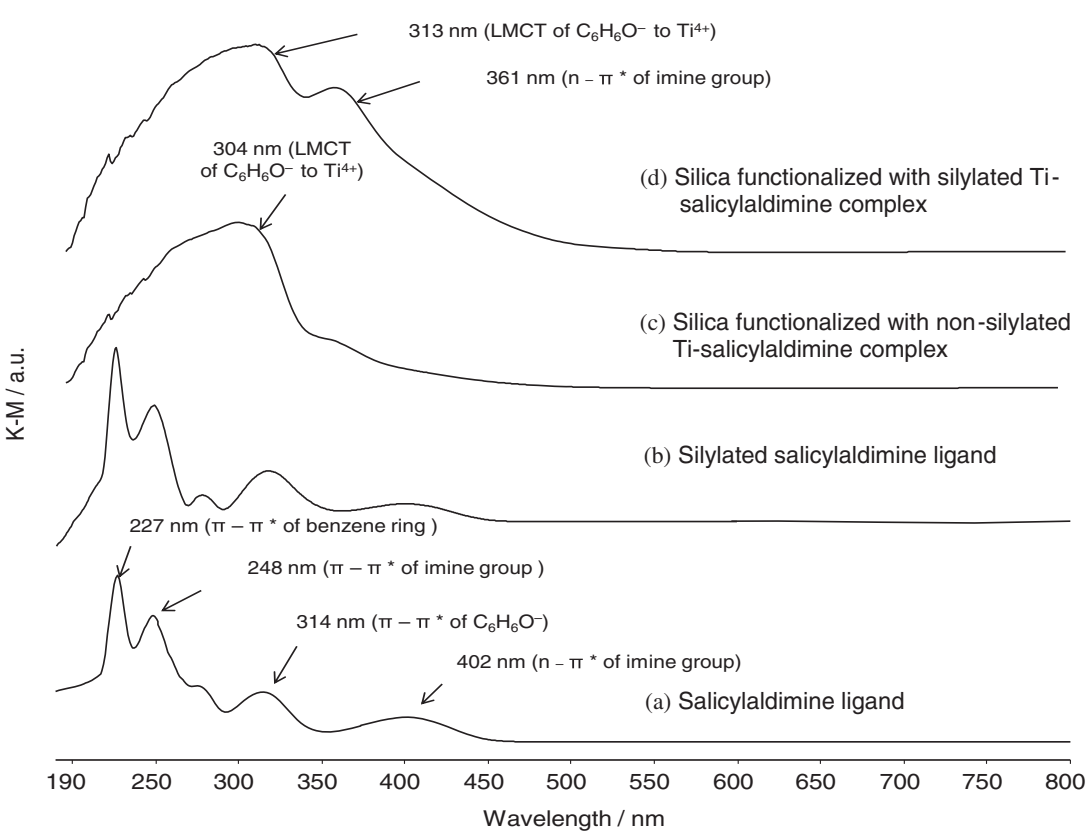

Figure 2. Diffuse reflectance UV-Vis spectra of (a) salicylaldimine ligand, (b) silylated salicylaldimine ligand, (c) silica functionalized with nonsilylated Ti-salicylaldimine complex and (d) silica functionalized with silylated Ti-salicylaldimine complex. 
compared to the silica functionalized non-silylated Tisalicyladimine complex. It is suggested that the silanol groups $(\mathrm{Si}-\mathrm{OH})$ have transformed into cross-linking Si-O-Si by OTMS, ${ }^{25}$ resulting in higher intensity of absorption bands in that region. ${ }^{28}$

\section{$3.3{ }^{29}$ Si solid state MAS NMR analysis}

Figure 3, (a-h) depicts the ${ }^{29} \mathrm{Si}$ solid state MAS NMR spectra of the silica functionalized with nonsilylated Ti-salicylaldimine complex and silylated Tisalicylaldimine complexes with Ti:OTMS molar ratios of 1:1/4 to $1: 4 .{ }^{29} \mathrm{Si}$ solid state MAS NMR spectroscopy is employed to examine the surface coverage and degree of cross-linking of the alkylsilyl groups. This analytical method can be a semi-quantitative tool in detecting the environmental alteration of Si from non-silylated to silylated Ti-salicylaldimine complex. Shoulder peaks with low density near $-57 \mathrm{ppm}$ were assigned to partial cross-linked $\mathrm{R}-\mathrm{Si}(\mathrm{OSi})_{2}(\mathrm{OH})$ and designated as $\mathrm{T}^{2}$ signals. ${ }^{7}$ The $\mathrm{T}^{3}$ signals at $-67 \mathrm{ppm}$ in the spectra showed the complete cross-linking of R-Si(OSi) . $_{4}$

It should be noted that the signal intensity of partial cross-linked $\mathrm{R}-\mathrm{Si}(\mathrm{OSi})_{2}(\mathrm{OH})$ (-57 ppm) was higher after the addition of OTMS to the complex (figure 3 (a) - (h)). This is caused by the increasing amount of $\mathrm{OH}$ groups $\left(-\mathrm{Si}(\mathrm{OSi})_{2}-\mathrm{OH}\right)$, which were not fully condensed to $\mathrm{R}-\mathrm{Si}(\mathrm{OSi})_{3}$. However, the amount of alkyl groups (carbon atoms) cannot be detected in this spectroscopy method, hence the amount of alkyl groups that were less than $\mathrm{Si}-\mathrm{OH}$ groups cannot be deduced. Thus, FTIR spectroscopy can be used as a supplementary tool to figure out whether the peak of alkyl groups increased with decreasing $\mathrm{Si}-\mathrm{OH}$ groups or vice-versa. The FTIR spectra in figure 1 showed increasing peak area for $\mathrm{sp}^{3} \mathrm{C}-\mathrm{H}$ stretching mode $\left(c a .2919 \mathrm{~cm}^{-1}\right.$ and $2850 \mathrm{~cm}^{-1}$ ) and decreasing peak area for $\mathrm{Si}-\mathrm{OH}$ band with increasing amount of OTMS.

When the peak area of $\mathrm{T}^{2}$ was compared to the peak area of $\mathrm{T}^{3}$, it can be seen that $\mathrm{T}^{2}$ has a smaller peak area. This indicates that the hydrolysis and condensation of OTMS and 3-aminopropyltrimethoxysilane have been completed with the presence of $\mathrm{R}-\mathrm{Si}(\mathrm{OSi})_{3}$ cross-linking at signal $\mathrm{T}^{3}$.

\subsection{SEM analysis}

Figure 4 displays that morphology of the two types of catalysts. Figures 4(a) and (b) are images of silica functionalized with non-silylated Ti-salicyladimine complex under magnification of x10000 and magnification of $\mathrm{x} 20000$, respectively, while figures 4(c) and
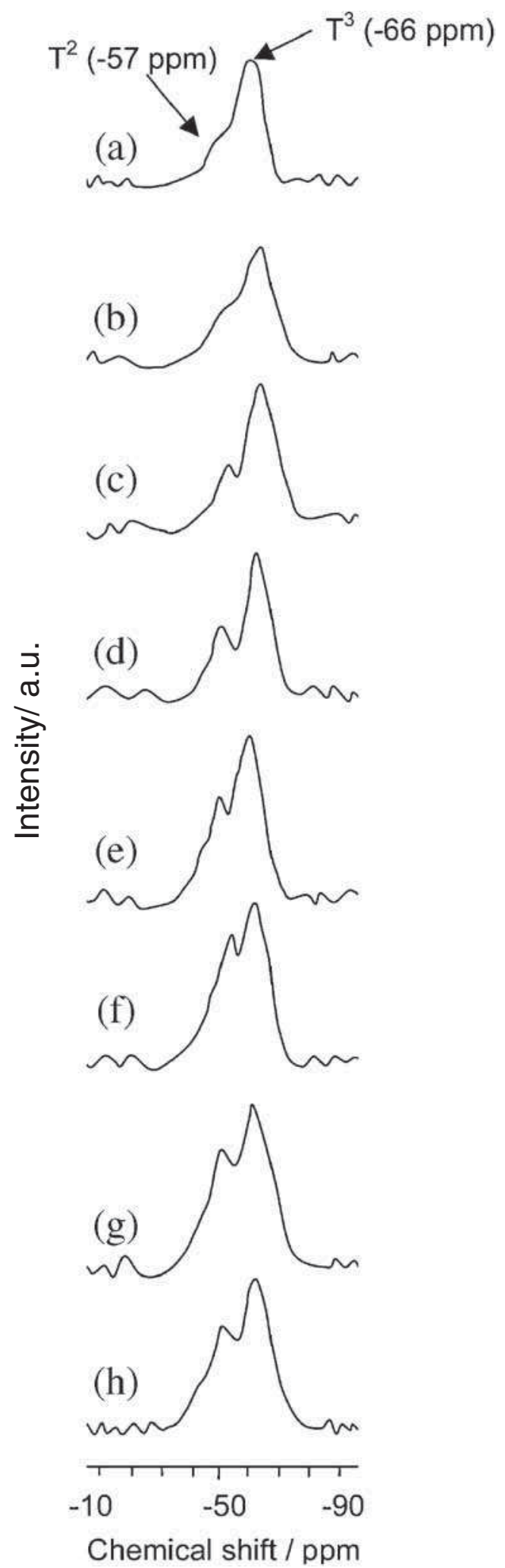

Figure 3. ${ }^{29} \mathrm{Si}$ solid state MAS NMR spectra of (a) silica functionalized with Ti-salicylaldimine complex, (b) silica functionalized with Ti-salicylaldimine complex with Ti:OTMS molar ratios of (b) $1: 1 / 4$, (c) $1: 1 / 3$, (d) $1: 1 / 2$, (e) $1: 1$, (f) $1: 2$, (g) $1: 3$, and (h) $1: 4$. 

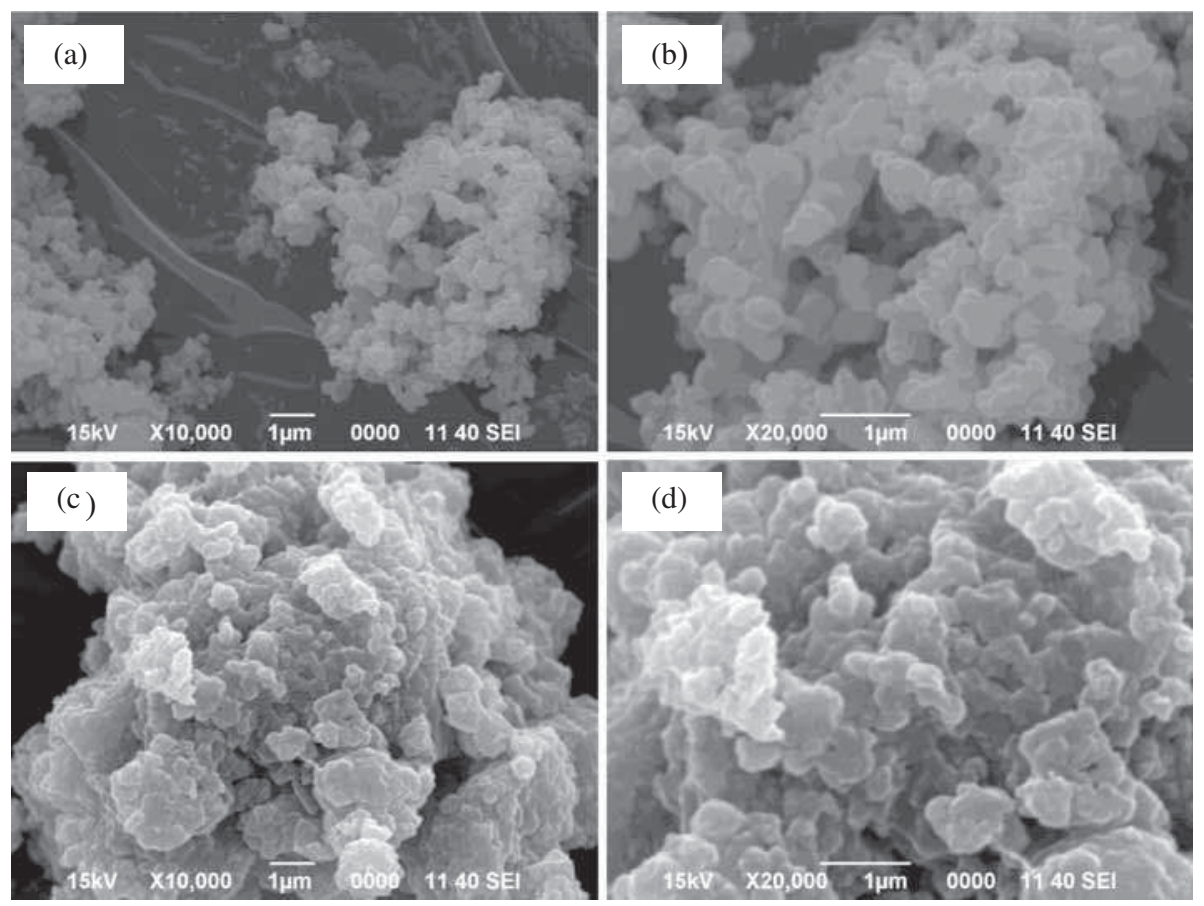

Figure 4. SEM micrographs of silica functionalized with Ti-salicylaldimine complex with (a) magnification of x10000, (b) magnification of x20000, (c) silylation of OTMS under magnification of x10000, and (d) silylation of OTMS under magnification of $\mathrm{x} 20000$.

(d) are images of silica functionalized with silylated Tisalicyladimine complex under magnification of x 10000 and magnification of $\mathrm{x} 20000$, respectively. It can be seen that the catalysts are amorphous or in disordered structure, which might be due to the route of synthesis. The main difference between the non-silylated and silylated catalysts is that the latter one showed obvious agglomeration in the presence of OTMS.

\subsection{TG analysis}

The thermogravimetric curves in figure 5 show the difference in mass loss for the silica functionalized with non-silylated Ti-salicyladimine complex and silylated Ti-salicylaldimine complex. For silica functionalized with non-silylated Ti-salicylaldimine complex, there are two steps involved: first, the mass loss of adsorbed water happened between $0-110^{\circ} \mathrm{C}$; while the second step is the mass loss of ligand organic moiety at the temperature between $300-700^{\circ} \mathrm{C}$.

For silica functionalized with silylated Tisalicyladimine complex with Ti/OTMS molar ratio of $1: 1 / 4,1: 1$, and 1:4, three steps are involved. The mass decomposition of the first two steps was same as the silica functionalized with non-silylated Ti-salicylaldimine complex, i.e. water desorption and ligand organic moiety decomposition. The third step happened at the temperature of $450-700^{\circ} \mathrm{C}$, owing to the mass loss of alkylsilyl groups. It is observed that the highest amount of alkylsilyl groups (Ti:OTMS $=1: 4$ ) contributed to the biggest mass loss, followed by the molar ratio of Ti:OTMS with 1:1 and the least mass loss is shown by the catalyst with the Ti:OTMS molar ratio of $1: 1 / 4$.

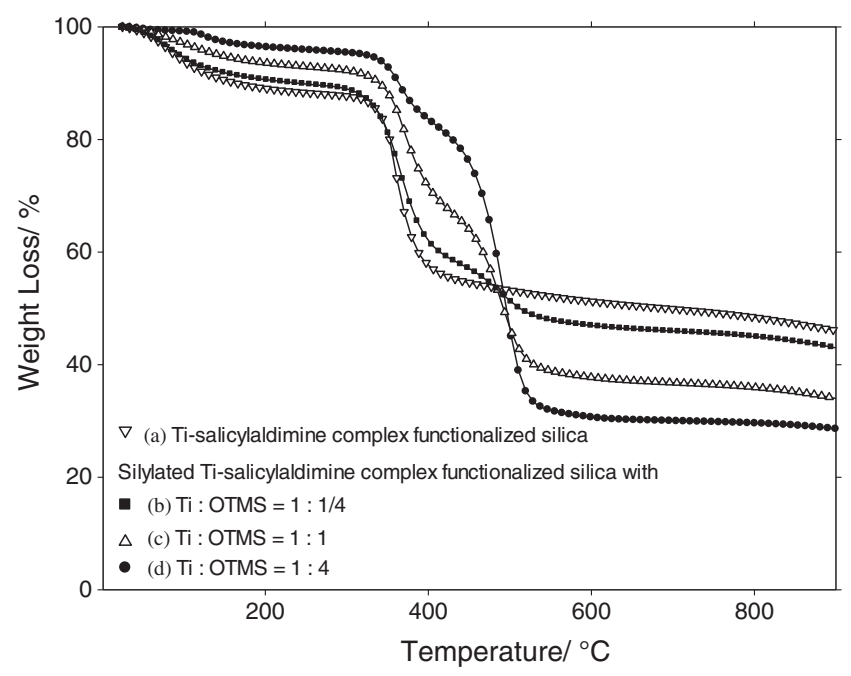

Figure 5. Thermogravimetric curves of (a) silica functionalized with Ti-salicylaldimine complex, silica functionalized with silylated Ti-salicyladimine complex with Ti/OTMS molar ratio of (b) 1:1/4, (c) 1:1, and (d) 1:4. 


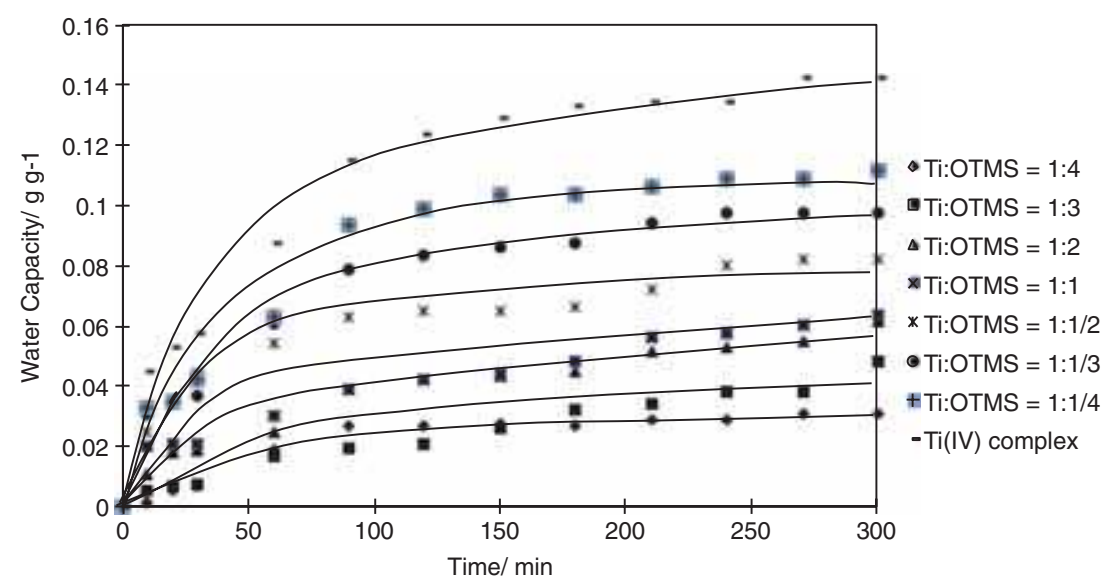

Figure 6. Water adsorption capacities $\left(\mathrm{mg} \mathrm{g}^{-1}\right)$ of silica functionalized with silylated and non-silylated Ti-salicylaldimine complex functionalized silica.

For the first step in the TG analysis, i.e. water desorption, it is clearly seen that the least water content adsorbed as shown by silica functionalized with silylated Ti-salicyladimine complex with Ti/OTMS molar ratio 1:4. This is attributed to its higher degree of hydrophobicity compared to the others. On the contrary, non-silica functionalized with silylated Tisalicyladimine complex showed the highest water content. The results of TG analysis are in correlation with the water adsorption analysis, which will be explained in section 3.6.

\subsection{Water adsorption analysis}

Figure 6 represents the results from the water adsorption capacity experiment carried out for $5 \mathrm{~h}$ by measuring the weight gained by the catalysts every $30 \mathrm{~min}$. It is interesting that Langmuir isotherm with first order kinetic reaction is observed. This shows that the water molecules adsorbed to a maximum of one monolayer on the solid surface with the rate constant, $k$, of $2.0 \mathrm{~h}^{-1}$. The kinetic rate obtained for the first $30 \mathrm{~min}$ is $2.32 \mathrm{X}$ $10^{-2} \mathrm{~g} \mathrm{~h}^{-1}$ for silica functionalized with non-silylated Ti-salicyladimine complex and $2.80 \times 10^{-3} \mathrm{~g} \mathrm{~h}^{-1}$ for silica functionalized with silylated Ti-salicylaldimine complex. This indicates that the non-silylated catalyst possesses higher water adsorption capacity and faster kinetics in reaction as compared to the silylated catalysts.

As shown in figure 6 and table 2, silica functionalized with non-silylated Ti-salicyladimine complex shows the highest water adsorption capacity, i.e. $142.00 \mathrm{mg} \mathrm{g}^{-1}$, and this implies that the complex functionalized silica was highly hydrophilic. This is in agreement with the FTIR spectra, where polar Si-OH groups appeared with the highest intensity, in comparison to those of the silica functionalized with silylated Ti-salicyladimine complex.

As reported in table 2, the water adsorption capacities $\left(\mathrm{mg} \mathrm{g}^{-1}\right)$ of the silylated samples gradually decreased with increasing amount of OTMS. Thus, it is evident that the hydrophobicity of these catalysts increased. It is observed that the water capacities of all the samples gradually became constant and this was attributed to water saturation on the superficial silanol groups. In order to investigate the effectiveness of the silylated and non-silylated Ti-salicyladimine complexas catalysts, the catalytic activity of these catalysts were tested out in an aqueous-organic-solid biphasic system. The comparison of the catalytic performances is made based on the introduction of octadecylsilyl groups $\left(\mathrm{C}_{18} \mathrm{H}_{37} \mathrm{Si}(\mathrm{OSi})_{\mathrm{n}}-\right)$ to these complexes, which is predicted to show significant improvement in the catalytic activity in the oxidation of cyclohexene and limonene by aqueous $\mathrm{H}_{2} \mathrm{O}_{2}$.

Table 2. Water adsorption capacities $\left(\mathrm{mg} \mathrm{g}^{-1}\right)$ of various Ti:OTMS molar ratios of OTMS silylation on Tisalicylaldimine complex.

Molar ratio of silylated

Ti-salicylaldimine complex

functionalized silica

Water adsorption

Ti:OTMS $=1: 4$ capacity $\left(\mathrm{mg} \mathrm{g}^{-1}\right)$

Ti:OTMS $=1: 3$

31.00

Ti:OTMS $=1: 2$

48.50

Ti:OTMS $=1: 1$

62.00

Ti:OTMS $=1: 1 / 2$

64.50

82.00

Ti:OTMS $=1: 1 / 3$

97.00

Ti:OTMS $=1: 1 / 4$

111.50

Ti:OTMS $=1: 0$ 


\subsection{Catalytic activity}

In this research, carveol and carvone are the main products in the oxidation of limonene by aqueous $\mathrm{H}_{2} \mathrm{O}_{2}$, as these two products favour the free radical allylic auto-oxidation reaction pathway. The plausible reaction pathway is shown in figure 7. Hydroxyl radicals $\left(\mathrm{OH}^{\bullet}\right)$, either from $\mathrm{H}_{2} \mathrm{O}_{2}$ or $\mathrm{H}_{2} \mathrm{O}$ after their interaction with $\mathrm{Ti}(\mathrm{IV})$ active sites, initiate limonene at the allylic carbon atom $\left(\mathrm{sp}^{3}\right.$-hybridized carbon in $\mathrm{CH}_{2} \mathrm{CH}-$ $\mathrm{CH}_{3}$ branch chain), which is stabilized by the resonance structure and a vinylic carbon ( $\mathrm{sp}^{2}$-hybridized carbon in $\mathrm{CH}_{2} \mathrm{CH}-\mathrm{CH}_{3}$ branch chain). Then, it is oxidized by the dissolved oxygen in solution to form limonene peroxo intermediate. ${ }^{29}$ This limonene peroxo intermediate can react with the limonene substrate to produce limonene hydroperoxide, which then undergoes rearrangement to generate carveol and carvone. The formation of carvone might be due to the water molecules that extracted the hydroxide ion of the limonene hydroperoxide. Hence, the negative charge on the oxygen in $\mathrm{C}-\mathrm{O}$ delocalizes to generate $\mathrm{C}=\mathrm{O}$. Meanwhile, the generation of carveol is attributed to the hydrogen addition process by the water molecules to the ketone group from carvone.

It is suggested that the formation of [Ti(salicylaldimine) $\mathrm{SO}_{4}$ complex in silica functionalized with Tisalicylaldimine complex has taken place. Imine group $(\mathrm{C}=\mathrm{N})$ is neutral and contains lone pair electrons, which can coordinate with $\mathrm{Ti}^{4+}$ by dative covalent bond. Meanwhile, two moles of $\mathrm{C}_{6} \mathrm{H}_{6} \mathrm{O}^{-}$bearing anionic oxygen ions can reduce the $\mathrm{Ti}^{4+}$ to $\mathrm{Ti}^{2+}$. Thus, the cationic complex can be formed, before being neutralized by one

(a) Initiation Steps

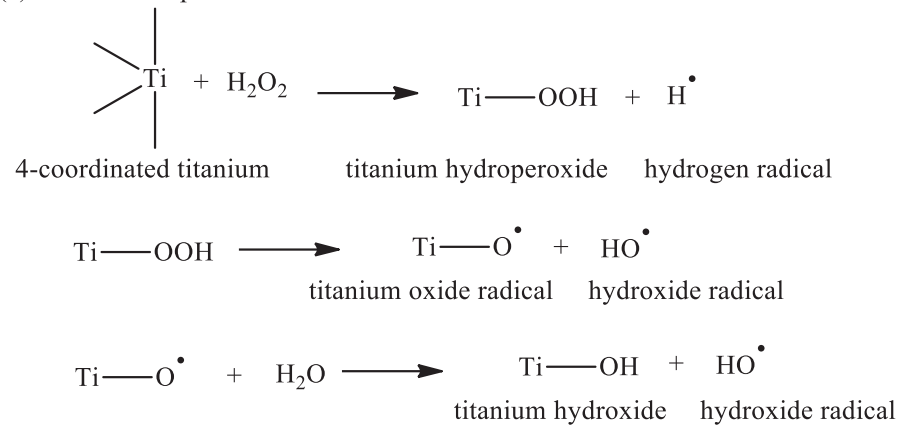

(b) Propagation and Termination Steps

(i)

<smiles>C=C(C)C1CC=C(C)C(OOC)C1</smiles><smiles>C=C(C)C1CC=C(C)CC1</smiles><smiles>CC1CCCC1</smiles><smiles>C=C(C)C1CC=C(C)C(OO)C1</smiles>

limonene hydroperoxide<smiles>C=C(C)C1CC=C(C)C(=O)C1</smiles>

carvone<smiles>C=C(C)C1CC=C(C)C(O)C1</smiles>

carveol

(c)

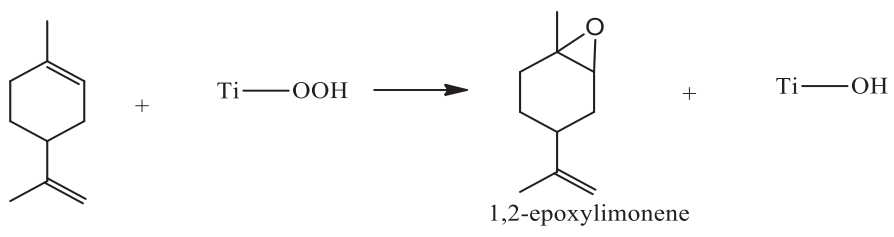

Figure 7. The plausible mechanistic pathway for limonene conversion. 
mole of $\mathrm{SO}_{4}^{2-}$ ion to form a neutral complex, as shown in the equation below.

2 (salicylaldimine $)^{-}+\mathrm{Ti}^{4+}+\mathrm{SO}_{4}^{2-} \longrightarrow\left[\mathrm{Ti}(\text { salicylaldimine })_{2}\right] \mathrm{SO}_{4}$

The catalytic activity of silica functionalized with non-silylated and silylated Ti-salicyladimine complex was carried out in the oxidation of limonene by aqueous $\mathrm{H}_{2} \mathrm{O}_{2}$. The highlight of this catalyst is that silica functionalized with non-silylated Ti-salicyladimine complex is in solid form without post-grafting onto any inorganic support. It is actually unexpected because there is usually a need to anchor metal complexes onto inorganic supports to form heterogeneous catalyst. This phenomenon might be due to the use of $24 \%$ Ti(IV) sulphate solution which contains high amount of water and thus the water assisted in complete hydrolysis and condensation reactions among methoxysilyl groups themselves, to form siloxane linkages. Therefore, solid silica functionalized with Ti-salicylaldimine complex was produced.

Silica functionalized with non-silylated and silylated Ti-salicyladimine complex were investigated as catalysts in the oxidation of limonene by aqueous $\mathrm{H}_{2} \mathrm{O}_{2}$. Figure 8 shows the hydrophobic effect towards the turnover number (TON) for $\mathrm{Ti}$ in the series of catalysts prepared. It was observed that the products from the oxidation of limonene by aqueous hydrogen peroxide $\left(\mathrm{H}_{2} \mathrm{O}_{2}\right)$, such as 1,2-epoxylimonene, 8,9epoxylimonene, carveol and carvone, were in accordance with literature reports. ${ }^{30}$ The silica functionalized with non-silylated Ti-salicyladimine complexhas the lowest TON (20) compared to that of the analogous silylated Ti-salicylaldimine complexes. This is due to the presence of a large number of silanol groups, as proven in the FTIR spectra (figure 1). These silanol groups can easily attract water molecules, which can suppress the active sites of $\mathrm{Ti}(\mathrm{IV})$. Moreover, it is also probably due to the polar products, such as epoxides, which have better interaction with polar surface of nonsilylated Ti-salicylaldimine complex functionalized silica. This can lead to the blocking of the transition metal active sites, thus hindering the oxidation. ${ }^{25}$

When the water adsorption capacity decreased, the TON for Ti increased, as shown in figure 8. The highest degree of hydrophobicity has contributed to highest TON of $\mathrm{Ti}$ (110) with conversion percentage of $61 \%$. The conversion increased by around $26 \%$ as compared to those shown by silica functionalized with nonsilylated Ti-salicylaldimine complex. This is due to the successful substitution of Si-OH by OTMS. Higher degree of hydrophobicity (lower water adsorption

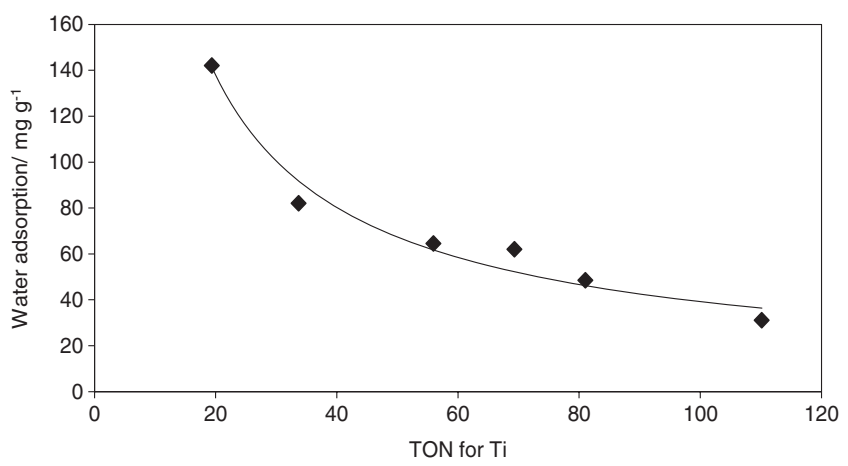

Figure 8. The effect of hydrophobicity in silica functionalized with silylated Ti-salicyladimine complex on the turnover number for $\mathrm{Ti}$ (TON) in the oxidation of limonene by aqueous hydrogen peroxide.

capacity) could result in decreasing number of $\mathrm{Si}-\mathrm{OH}$ and this suppresses the adsorption of water molecules onto the active sites of Ti. This is owing to the weak acid characteristic of $\mathrm{Si}-\mathrm{OH}$ and $\mathrm{H}_{2} \mathrm{O}$, that is able to induce the oxirane ring opening, before forming glycols, which will then lead to the deactivation of the catalysts. ${ }^{25}$

It should be highlighted that the TON for Ti gradually became constant as the water adsorption capacity decreased. This phenomenon could probably be caused by the concentration of OTMS that reached the maximum coverage of the sureface of the complex. Introducing excess OTMS onto the catalyst may hydrolyze and form octadecylsilyl groups, which comprised of $\mathrm{C}_{18} \mathrm{H}_{37}-$ and -Si-OH. As a result, limonene and $\mathrm{H}_{2} \mathrm{O}_{2}$ will be attracted to the hydrolyzed octadecylsilyl groups and this may reduce the catalytic activity of the catalyst in the reaction.

Figure 9 shows the conversion of limonene and selectivity of carveol, carvone, and other products after the catalytic reaction. The selectivity of other unidentified products (may include 1,2-epoxylimonene, 8,9-epoxylimonene, diepoxy product, and glycols) decreased with increasing hydrophobicity.

Table 3 shows the concentration of Ti in silica functionalized with silylated Ti-salicyladimine complex in the oxidation of limonene. It is seen that Ti content in $50 \mathrm{mg}$ of catalysts has slightly decreased but it did not affect the results of TON for Ti and product yields. This indicates that the hydrophobicity from the alkylsilyl groups plays a significant role in the product yields.

It is found that most of the previous studies used cyclohexene as the reactant in the oxidation reactions for alkylsilylated catalysts. Despite this, a few reports can be obtained for the elucidation of the silylation effect on the catalytic activity in the limonene oxidation. ${ }^{31,32}$ The catalyst's structure design (Ti-salicylaldimine complex functionalized 


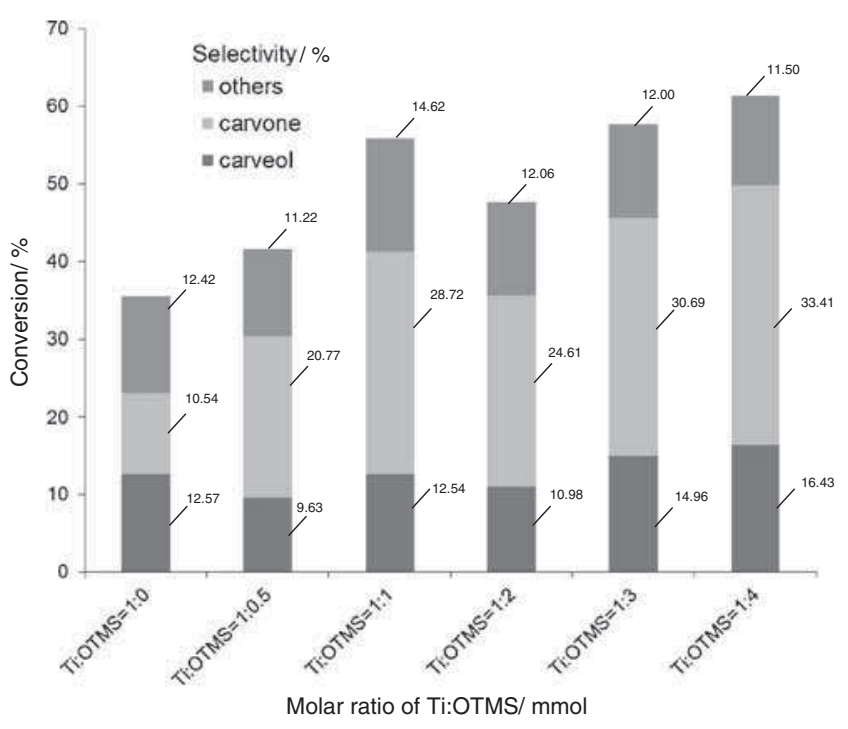

Figure 9. The conversion of limonene and the yields of carvone, carveol and other products after the catalytic reaction. The reactions were carried out at $70^{\circ} \mathrm{C}$ for $22 \mathrm{~h}$ with (R)-(+)-Limonene (5 mmol, $0.83 \mathrm{~mL}$ ), $30 \%$ aqueous $\mathrm{H}_{2} \mathrm{O}_{2}$ $(10 \mathrm{mmol}, 0.38 \mathrm{~mL})$ and catalyst $(50 \mathrm{mg})$ under stirring conditions.

silica) for this research is essentially quite different from those reported, i.e. Ti-MCM-41. Silylated TiMCM-41 achieved a better performance (79-92\% conversion) compared to silica functionalized with silylated Ti-salicyladimine complex (61\% conversion) in limonene oxidation. It might be assigned to two possible reasons, which will be explained in the following paragraphs.

First, Ti-MCM-41 contained Ti incorporated within the framework of the hexagonal mesoporous solid powder, while silica functionalized with Ti-salicylaldimine complex is disordered and non-porous solid with $\mathrm{Ti}$ coordinated to ligand. Limonene is a small, aromatic molecule which can adsorb, react and desorb on $\mathrm{Ti}$ active sites on the surface of MCM-41 or diffuse through the pores of MCM-41, to reach the Ti active sites inside the porous structure. This structure could provide a higher surface area for the reaction to occur and hence, the higher catalytic activity. Silica functionalized with Ti-salicylaldimine complex possesses lower surface area as it is nonporous and the reaction can only take place on the outer surface of the catalyst.

Second, the silylating agent used in the previous researches ${ }^{31,32}$ was hexamethyldisilazane (HDMS) which is a short alkylsilyl chain, while in this reaserch octadecyltrimethoxysilane (OTMS), which is a long alkylsilyl chain, was employed. The long alkylsilyl groups might have caused steric hindrance to the diffusion of limonene to the Ti active sites. On the contrary, limonene, which is branched, can come into contact with the Ti active sites more easily when shorter alkylsilyl groups on the catalyst's internal and outer surface were used.

Thus, the catalytic activity of the catalyst in this research is slightly lower than those previously reported due to the difference in structure design (surface area), types of silylating agent and substrates used. However, it was observed that in both the catalytic systems, Ti-MCM-41 or silica functionalized with Tisalicylaldimine complex, the selectivity increased with increasing concentration of silylating agent added.

Figure 10 displays the stability results of the silylated catalysts that were obtained by conducting the reusability testing with the same reaction condition. It was observed that the limonene conversion of the silica functionalized with non-silylated Ti-salicyladimine complex decreased tremendously for the second run due to the adsorption of the water molecules on the Ti active sites to form Ti-OH or opening of the epoxy ring to form glycols. On the contrary, those of silica functionalized with silylated Ti-salicyladimine complex

Table 3. The concentration of $\mathrm{Ti}$ in silica functionalized with silylated Tisalicyladimine complex catalysts.

\begin{tabular}{lcccr}
\hline $\begin{array}{l}\text { Silylated Ti-salicylaldimine } \\
\text { complex functionalized } \\
\text { silica }^{\text {a }}\end{array}$ & $\begin{array}{c}\text { Weight of } \\
\text { catalysts } \\
(\mathrm{mg})\end{array}$ & $\begin{array}{c}\text { Ti in } 50 \mathrm{mg} \\
\text { of catalysts } \\
(\mathrm{mmol})\end{array}$ & $\begin{array}{c}\text { Products } \\
(\mathrm{mmol})\end{array}$ & $\begin{array}{c}\text { TON for } \\
\mathrm{Ti}\end{array}$ \\
\hline Ti:OTMS $=1: 0$ & 582.90 & 0.09 & 1.78 & 19.00 \\
Ti:OTMS $=1: 0.5$ & 862.50 & 0.06 & 2.09 & 34.00 \\
Ti:OTMS $=1: 1$ & 1069.30 & 0.05 & 2.80 & 56.00 \\
Ti:OTMS $=1: 2$ & 1558.80 & 0.03 & 2.38 & 69.00 \\
Ti:OTMS $=1: 3$ & 1505.20 & 0.04 & 2.88 & 81.00 \\
Ti:OTMS $=1: 4$ & 1919.50 & 0.03 & 3.07 & 110.00 \\
\hline
\end{tabular}

${ }^{\mathrm{a}}$ The concentration of $\mathrm{Ti}$ was calculated based on the amount of Ti(IV) sulphate added in the synthesis of the catalysts. A constant concentration of Ti(IV) sulphate $(1.07 \mathrm{mmol})$ was used throughout the synthesis of the catalysts. 


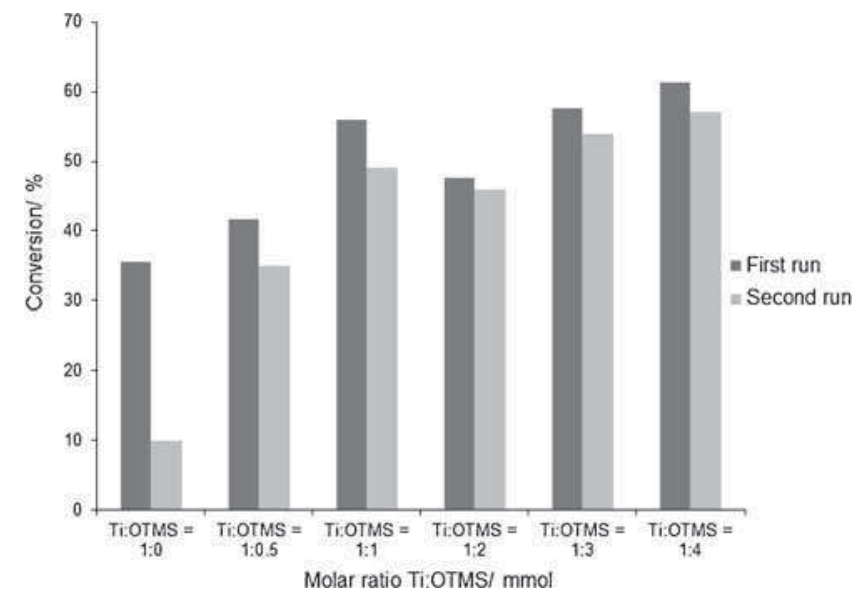

Figure 10. Comparison of the reusability of $(\mathrm{R})-(+)-$ Limonene for $1^{\text {st }}$ and $2^{\text {nd }}$ run in the oxidation of limonene by aqueous $\mathrm{H}_{2} \mathrm{O}_{2}$ at $70^{\circ} \mathrm{C}$ for $22 \mathrm{~h}$.

remained almost the same, with only slight decrease in the range of $2-6 \%$. Therefore, it is shown that the hydrophobic surface of these catalysts has successfully repelled the adsorption of water molecules on the $\mathrm{Ti}$ active sites to prevent deactivation.

\section{Conclusions}

The catalytic activity of silica functionalized with Tisalicylaldimine complex in the oxidation of limonene was significantly improved by introducing hydrophobicity on the catalyst surface. These results strong depended on the amount of octadecylsilyl organic moiety immobilized on the catalyst. It was observed that the TON for Ti increased when the rate of water adsorption decreased. It is significant to conclude that the improved catalytic activity of the catalysts might due to the hydrophobic surface. The decrease in polarity of silanol groups and suppression of water molecules can be an idea to further improve the catalyst's design and productivity in various applications for future works.

\section{Supplementary Information}

All additional information pertaining to characterization of the complexes using FTIR peak area data (table 1), FTIR spectra (figure S1), electronic spectra (figure S2), thermogravimetric graph (figure S3) and GC chromatogram (figure S4) are given in the supporting information. Supplementary Information is available at www.ias.ac.in/chemsci.

\section{Acknowledgments}

The authors gratefully acknowledge funding from the Ministry of Higher Education (MOHE), Malaysia through Fundamental Research Grant Scheme (FRGS) and Research University Grant (GUP), Postdoctoral Fellowship Scheme by Research Management Centre (RMC), Development of Low Carbon Scenarios for Asia Region, Universiti Teknologi Malaysia.

\section{References}

1. Notari B 1993 Catal. Today 18163

2. Elías V R, Crivello M E, Herrero E R, Casuscelli S G and Eimer G A 2009 Ind. Eng. Chem. Res. 489076

3. Nur H, Ikeda S and Ohtani B 2001 J. Catal. 204402

4. Chu H, Wan Y and Zhao D 2009 Catal. Today 14819

5. Kholdeeva O A and Maksimovskaya R I 2007 J. Mol. Catal. A Chem. 2627

6. Lin K, Pescarmona P P, Vandepitte H, Liang D, Tendeloo G V and Jacobs P A 2008 J. Catal. 25464

7. Nur H, Prasetyoko D, Ramli Z and Endud S 2004 Catal. Commun. $\mathbf{5} 725$

8. Nur H, Hau N Y, Misnon I I, Hamdan H and Muhid M N M 2006 Mater. Lett. 602274

9. Prasetyoko D, Ramli Z, Endud S and Nur H 2005 Mater. Chem. Phys. 93

10. Lin K, Pescarmona P P, Houthoofd K, Liang D, Tendeloo G V and Jacobs P A 2009 J. Catal. 26375

11. Capel-Sanchez M C, Campos-Martin J M and Fierro J L G 2010 Catal. Today 158103

12. Corma A, Domine M, Gaona A J, Jorda L J, Navarro T M, Rey F, Perez-Pariente J, Tsuji J, McCulloch B and Nemeth T L 1998 Chem. Commun. 202211

13. Corma A, Jordá J L, Navarro M T, Pérez-Pariente J, Rey F and Tsuji J 2000 Stud. Surf. Sci. Catal. 129169

14. Bu J and Rhee H-K 2000 Catal. Lett. 65141

15. Igarashi N, Hashimoto $\mathrm{K}$ and Tatsumi T 2007 Microporous Mesoporous Mater. 104269

16. Nur H, Hau N Y, Muhid M N M and Hamdan H 2004 Phys. J. IPS A7 0218

17. Peña M L, Dellarocca V, Rey F, Corma A, Coluccia S and Marchese L 2001 Microporous Mesoporous Mater. 44-45 345

18. Yuan L S, Razali R, Efendi J, Buang N A and Nur H 2013 Appl. Catal. A 460-461 21

19. Nakamoto K 1986 In Infrared and Raman spectra of organic and coordination compounds (4th ed.) (New York: John Wiley and Sons) p. 14

20. Yuan L S, Efendi J, Razali N S H and Nur H 2012 Catal. Commun. 2085

21. Mostafa S I, Ikeda S and Ohtani B 2005 J. Mol. Catal. A Chem. 225181

22. Roy G B 2009 Inorg. Chim. Acta 3621709

23. Hu C, Zhang W, Xu Y, Zhu H, Ren X, Lu C, Meng Q and Wang H 2001 Transit. Metal Chem. 26700

24. Luo Y, Lu G Z, Guo Y L and Wang Y S 2002 Catal. Commun. 3129

25. Yang Y, Guan J, Qiu P and Kan Q 2010 Appl. Surf. Sci. 2563346

26. İspir E 2009 Phosphorus Sulfur Silicon Relat. Elem. 184(12) 3160 
27. Parida K M, Sahoo M and Singha S 2010 J. Catal. 276 161

28. García-González C A, Sampaio da Sousa A R, Argemí A, López Periago A, Saurina J, Duarte C M M and Domingo C 2009 Int. J. Pharm. 382296

29. Cagnoli M V, Casuscelli S G, Alvarez A M, Bengoa J F, Gallegos N G, Samaniego N M, Crivello M E, Ghione G E, Pérez C F, Herrero E R and Marchetti S G 2005 Appl. Catal. A 287227
30. Saikia L, Srinivas D and Ratnasamy P 2006 Appl. Catal. A 309144

31. Guidotti M, Batonneau-Gener I, Gianotti E, Marchese L, Mignard S, Psaro R, Sgobba M and Ravasio N 2008 Microporous and Mesoporous Mater. 111 39

32. Cagnoli M V, Casuscelli S G, Alvarez A M, Bengoa J F, Gallegos N G, Crivello M E, Herrero E R and Marchetti S G 2005 Catal. Today 107-108 397 